\title{
Response to Letter Written by Shelly Cummings, MS, CGC of Myriad Genetics Laboratories, Inc. Regarding the Paper, "Racial and Ethnic Differences in Direct-to-Consumer Genetic Tests Awareness in HINTS 2007: Sociodemographic and Numeracy Correlates." J Genet Counsel (2012) 21:440-447
}

\author{
Aisha T. Langford • Ken Resnicow • J. Scott Roberts • \\ Brian J. Zikmund-Fisher
}

Received: 12 October 2012 / Accepted: 17 October 2012 /Published online: 26 October 2012

(C) National Society of Genetic Counselors, Inc. 2012

Thank you for your thoughtful comments and for taking time to read our paper. We agree that we should have been more explicit about distinguishing DTC advertising from testing when mentioning the Myriad BRCA testing advertisement campaign. We also agree that it is possible that some people could have responded affirmatively to the survey question about DTC test awareness when it was really DTC advertising that they were aware of. The HINTS 2007 question was worded as, "Genetic tests that analyze your DNA, diet, and lifestyle for potential health risks are currently being marketed by companies directly to consumers. Have you heard or read about these genetic tests?" As with any secondary data analysis, we cannot assess how respondents interpreted the question, nor can we distinguish how they specifically became aware of DTC genetic tests (e.g., from DTC advertising, healthcare providers, websites, family members or friends).

The goal of our study was not to estimate true awareness of DTC tests with high level precision but rather to look at racial/ethnic group differences on a DTC genetic test awareness item, while also evaluating numeracy correlates. As with all self-report items like those used in HINTS 2007, there is some level of measurement error. While such errors likely affect the accuracy of our estimates of the overall prevalence of consumer awareness of DTC genetic testing products, we find it difficult to see how this issue would materially change the interaction with numeracy that is the core of our research findings.

We note in our paper that there is no consensus in the field on the best way to present health information in the context of genetic tests (both DTC and clinical included). We also outline several areas of potential research such as communications training, different ways to present numbers, and screening tools to assess patient preferences for health information. More research in these areas may help support informed decision making among consumers and equip genetic counselors with better tools to use with patients.

A. T. Langford $(\bowtie) \cdot K$. Resnicow $\cdot$ J. S. Roberts $\cdot$

B. J. Zikmund-Fisher

University of Michigan School of Public Health,

Ann Arbor, MI, USA

e-mail: alangfor@umich.edu 